\section{Targeting acute myeloid leukemia with TP53-independent vosaroxin}

\author{
Christopher B Benton' \& Farhad Ravandi ${ }^{*, 1}$
}

Vosaroxin is a quinolone compound that intercalates DNA and induces TP53-independent apoptosis, demonstrating activity against acute myeloid leukemia (AML) in Phase I-III trials. Here, we examine vosaroxin's mechanism of action and pharmacology, and we review its use in AML to date, focusing on details of individual clinical trials. Most recently, when combined with cytarabine in a randomized Phase III trial (VALOR), vosaroxin improved outcomes versus cytarabine alone for relapsed/refractory AML in patients older than 60 years and for patients in early relapse. We consider its continued role in the context of a multifaceted strategy against $A M L$, including its current use in clinical trials. Prospective use will define its role in the evolving landscape of AML therapy.

First draft submitted: 24 June 2016; Accepted for publication: 17 August 2016; Published online: 12 September 2016

Quinolone-related compounds were noted to be potential antitumor candidates due to their activity inhibiting eukaryotic topoisomerase II, a common target of other cancer chemotherapies [1]. Vosaroxin (previously voreloxin) is a quinolone naphthyridine analog, and it intercalates into DNA to inhibit topoisomerase II in replicating cells [2]. Because of its favorable toxicity profile and activity against leukemia in preclinical studies [3], it has been developed as a therapy for acute myeloid leukemia (AML), a difficult-to-treat and heterogeneous disease that is diagnosed in nearly 20,000 patients in the USA annually [4]. New treatments have been sought because outcomes for many AML patients are particularly unfavorable, especially in certain subgroups such as older patients [5]. Vosaroxin has completed Phase I-III studies for AML. We review its role in AML from past studies and as recently defined by the VALOR trial, a very large multicenter randomized trial, which allowed informative subset analyses [6].

\section{Pharmacology of vosaroxin}

Structural characteristics that define the quinolone class of compounds allow them to intercalate DNA most effectively during the G2/M cell division stage, inducing double-strand breaks in GC-rich regions. The full extent of vosaroxin's mechanism of action is reviewed in Figure 1 [2,7]. Intercalation is toxic to topoisomerase II by way of a different mechanism than other topoisomerase II inhibitors, such as etoposide and anthracyclines [2]. First, etoposide does not intercalate; instead, it induces DNA fragmentation that disrupts the action of topoisomerase II [2]. Although anthracycline antibiotics do intercalate DNA, they also generate significant reactive oxygen species, a property that is not shared by vosaroxin $[2,8]$. Additionally, the extent of DNA fragmentation and the homologous recombination-repair mechanisms triggered differ between vosaroxin and

'Department of Leukemia, The University of Texas MD Anderson Cancer Center, Houston, TX, USA

*Author for correspondence: fravandi@mdanderson.org

\section{KEYWORDS}

- acute myeloid leukemia

- antineoplastic agents

- intercalating agents

- prospective studies

- vosaroxin 


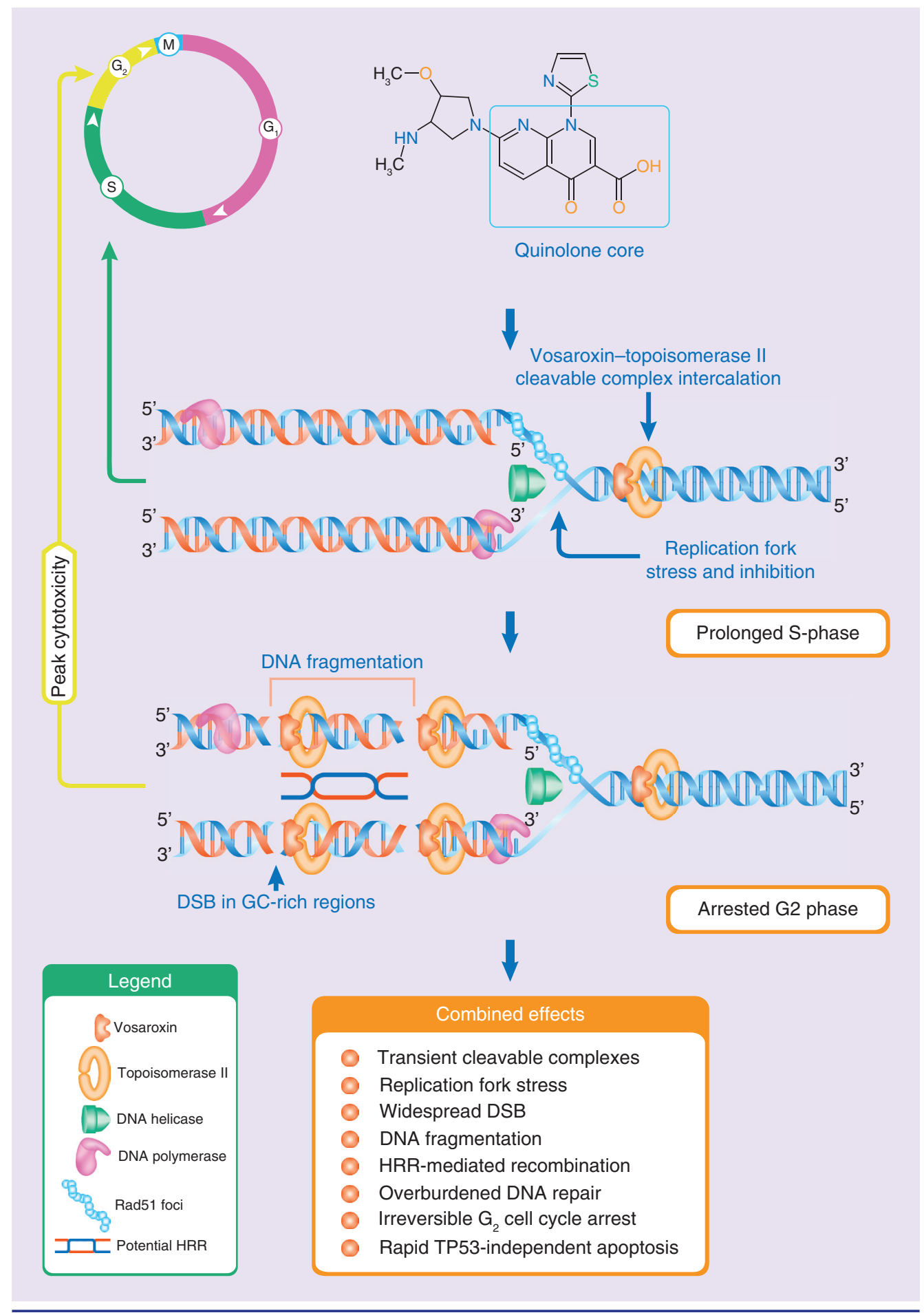

Figure 1. Vosaroxin mechanism of action. Vosaroxin acts on DNA during S-phase and G2/M-phase of the cell cycle. It combines with topoisomerase II and forms a transient cleavable complex that intercalates DNA due to its quinolone core. This induces replication fork stress and inhibition, and prolongs S-phase. More importantly, intercalation promotes DSBs in GC-rich regions resulting in DNA fragmentation. The process may also lead to HRR-mediated recombination, and overburdened DNA repair machinery. Ultimately, the combined effects of vosaroxin intercalation into DNA lead to cell-cycle inhibition and TP53-independent, proliferative acute myeloid leukemia cell apoptosis. DSB: Double-stranded break; HRR: Homologous recombination repair. 
anthracyclines [8]. Because of differences in their mechanisms of action, vosaroxin does not share the same degree of cardiac toxicity associated with anthracyclines [8]. Importantly, apoptosis induced by vosaroxin is TP53 mutation-status independent, and its mechanism bypasses the P-glycoprotein resistance pathway (a drug efflux mechanism and a known mechanism of AML resistance to chemotherapy [9]), making it an attractive drug for use in AML [10]. Vosaroxin was noted to cause marked myelotoxicity, and it synergized with cytarabine against myeloid leukemia in vitro and in vivo in preclinical studies [3,10-12,3].

Preclinical cross-species pharmacologic studies suggested a favorable metabolism profile for vosaroxin [11]. In pharmacokinetic studies, vosaroxin demonstrated a half-life of approximately $25 \mathrm{~h}$ [12]. Vosaroxin was metabolized predictably and similarly among patients of all ages, including those older than 65 years [12]. Because AML often afflicts older patients, vosaroxin's pharmacologic profile made it attractive for use in AML. Doselimiting toxicity was stomatitis, and there was a dose-dependent occurrence of febrile neutropenia and gastrointestinal toxicity [12]. Likewise, there was dose-dependent antileukemia activity, as measured by weekly time that vosaroxin concentration was above $1 \mu \mathrm{mol} / \mathrm{l}$, or total dose of vosaroxin administered [12]. An encouraging assessment based on these studies led to the use of vosaroxin on a number of protocols that enrolled adverse risk AML patients.

\section{Phase I \& II clinical trials}

Vosaroxin was first tested in Phase I and II clinical trials, and used to treat solid tumors $[13,14]$ and hematologic tumors including AML. Whether vosaroxin may be useful in other tumor types, such as small-cell lung cancer or ovarian cancer, has yet to undergo full evaluation $[13,15]$. Its use gained traction in leukemia, and Table 1 summarizes all reported clinical trials to date that have evaluated vosaroxin in AML. Early Phase I/II clinical trials were dosing studies that tested escalation and schedules [13,16-19,13]. The Phase II REVEAL-1 trial investigated vosaroxin in 113 adverse-risk AML patients $\geq 60$ years (median age was 75 years) and found a complete response/complete response with incomplete recovery of platelets (CR/CRp) rate of 35\% with a 1-year overall survival of 38\% [16]. Efficacy and mortality were comparable to historical data that likely included more favorable-risk patients [16,17-21]. Other Phase I/II trials allowed for a better understanding of dosing and toxicities; however, the smaller numbers of patients did not allow definitive subgroup analyses [16]. Given the comparable response to historical patients, favorable safety profile and data showing synergy with cytarabine, vosaroxin was further considered for use in larger Phase III combination trials. The relative dearth of standard-ofcare cytotoxic and therapeutic options available for use in adverse-risk AML led to a large collaborative effort to enroll significant numbers of patients to allow detailed subgroup assessments. Investigators hypothesized that older patients with AML and those with additional comorbidities may benefit from vosaroxin, which has good anti-AML activity, lower cardiac toxicity than anthracyclines and faster hematologic recovery compared with other cytotoxic agents [16].

\section{LI-1 \& VALOR}

Most recently, useful data matured from two trials, which together administered vosaroxin plus cytarabine to over 500 patients with AML aged over 60 years. One independent trial in the UK, the LI-1 (vosaroxin) trial (hereafter referred to as LI-1), sought to investigate single-agent and combinatorial strategies using drug-of-interest plus cytarabine; LI-1 tested vosaroxin in two separate randomized comparisons [19]. Patients enrolled were deemed unfit for intensive chemotherapy. In one head-to-head LI-1 trial, singleagent vosaroxin $\left(72 \mathrm{mg} / \mathrm{m}^{2}\right.$ intravenously [iv.] days 1,4$)$ was compared with single-agent lowdose cytarabine (20 mg subcutaneously [sc.], twice daily, days 1-10); in the second headto-head randomized comparison, vosaroxin $\left(72 \mathrm{mg} / \mathrm{m}^{2}\right.$ iv. days 1,4$)$ plus low-dose cytarabine (20 mg sc., twice daily, days 1-10) was compared with single-agent low-dose cytarabine $(20 \mathrm{mg}$ sc., twice daily, days 1-10). Both comparison trials were discontinued because a clinically and statistically relevant benefit was deemed unlikely. Notably, patients in the vosaroxin plus cytarabine group $(n=53)$ had the highest fraction of overall responses $(38 \%$, nonsignificant vs comparison group); however, this group also had increased early mortality, contributing to the lack of detectable benefit.

VALOR was a larger, industry-sponsored Phase III randomized trial that tested two arms: vosaroxin plus intermediate-dose cytarabine combination versus single-agent intermediatedose cytarabine (given with placebo). This 


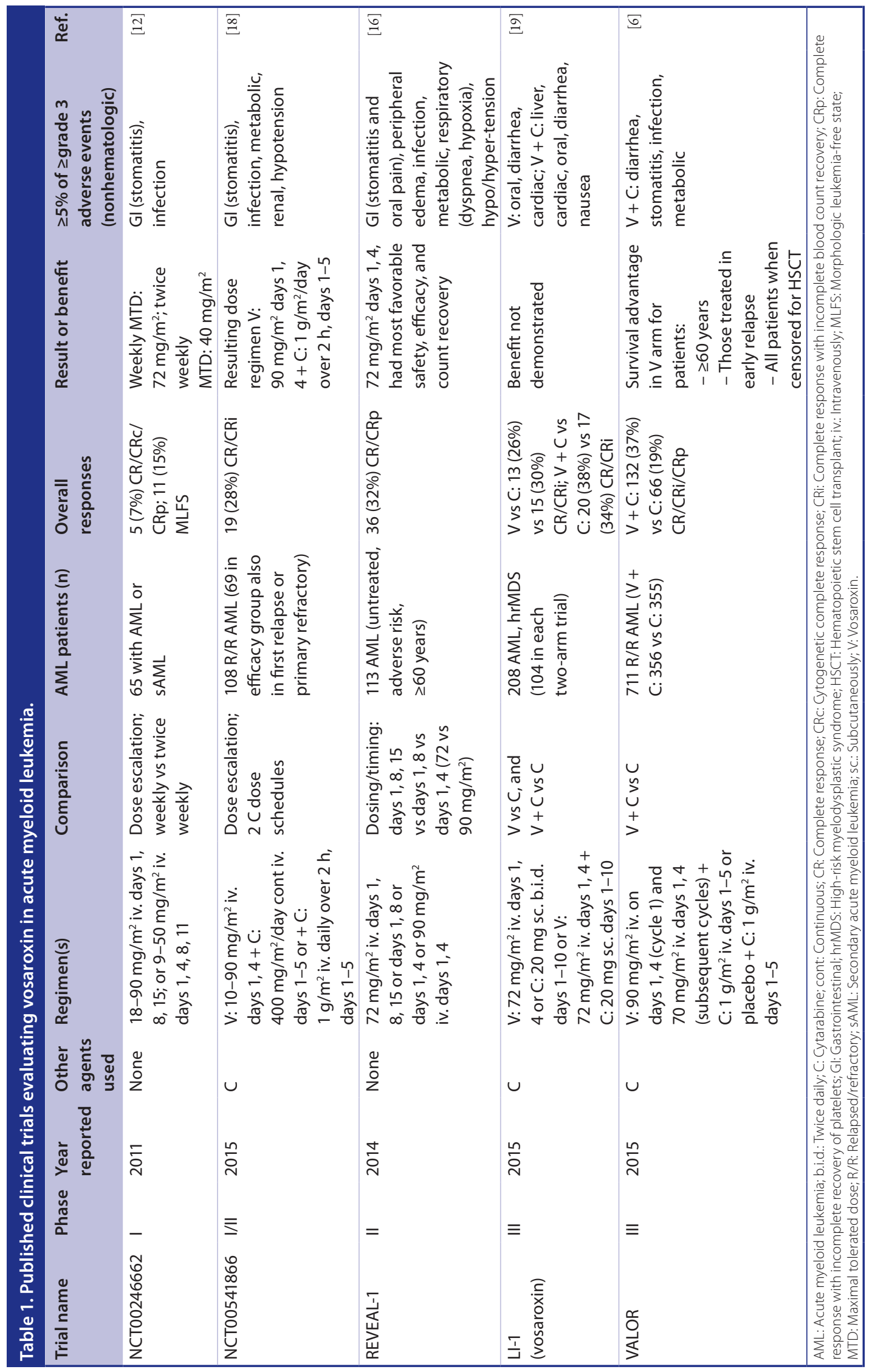


international multicenter effort was the largest of its kind for relapsed AML and evaluated 711 patients total from 101 institutions, randomizing relapsed/refractory AML patients to receive cytarabine alone $\left(1 \mathrm{~g} / \mathrm{m}^{2}\right.$ iv. days $\left.1-5\right)$ or cytarabine $\left(1 \mathrm{~g} / \mathrm{m}^{2}\right.$ iv. days $\left.1-5\right)$ plus vosaroxin $\left(90 \mathrm{mg} / \mathrm{m}^{2}\right.$ iv. days 1,4 [cycle 1] and $70 \mathrm{mg} / \mathrm{m}^{2}$ iv. days 1,4 [subsequent cycles]) [6]. All patients had previously received an anthracycline (or anthracenedione) plus cytarabine regimen, and they were in first relapse or had primary refractory disease. Patients were considered relapsed if they had reemergence of at least $5 \%$ leukemia blasts in the marrow or at least $1 \%$ blasts in peripheral blood at 90 days to 24 months after first complete remission (CR1) or CRp; and patients were considered refractory if they had persistent disease at least 28 days after initiation of induction, or relapse less than 90 days after CR1 or complete response with incomplete blood count recovery (CRi). The trial was double-blinded, placebocontrolled and used an adaptive design. Results, summarized in Table 1, showed a significantly increased overall response rate (ORR) in the arm receiving vosaroxin. Detailed subgroup analysis showed that certain patient groups benefited from the addition of vosaroxin to cytarabine, and were responsible for improved ORR.

There was a non- but near-significant overall survival benefit observed in all patients in the vosaroxin plus cytarabine cohort (median: 7.5 vs 6.1 months; $\mathrm{p}=0.061$ [unstratified logrank]; $\mathrm{p}=0.024$ [stratified log-rank]). A sensitivity analysis was performed on the survival data, censoring patients undergoing subsequent allogeneic stem-cell transplantation, and a survival advantage was found in the vosaroxin-treated arm (median: 6.7 vs 5.3 months; $\mathrm{p}=0.024$ [unstratified log-rank]; $\mathrm{p}=0.027$ [stratified log-rank]). Preplanned randomization-factor subgroup analyses were also performed. When patients were stratified by relapse status, a survival benefit in the vosaroxin arm was noted for those treated in early relapse (defined as occurring between 90 days and 12 months of preceding remission) with a median survival of 5.2 versus 6.7 months $(p=0.039)$. More significantly, when patients were stratified by age, those $\geq 60$ years were also noted to have a survival advantage if treated with vosaroxin plus cytarabine (median: 7.1 vs 5.0 months; $\mathrm{p}=0.003$ ). This group of patients often has particularly resistant disease and poorer overall prognosis. In total, the VALOR Phase III trial showed that the addition of vosaroxin led to improvement in survival for a specific subset of patients, comprised of those aged $\geq 60$ years with early relapsed or refractory AML.

Because the LI-1 and VALOR trials that investigated vosaroxin arrived at unique conclusions, a careful assessment of their differences is noteworthy (summarized in Table 2). First, the trials enrolled different patient populations and different numbers of patients. LI-1 enrolled patients unsuitable for intensive induction (total $n=208$ ), and VALOR only enrolled relapsed/refractory patients who had previously received an anthracycline (or anthracenedione) plus cytarabine regimen as treatment for AML (only one patient had received any anthracycline in LI-1; total $n=711$ ). Next, a smaller percentage of patients had Eastern Cooperative Oncology Group/WHO performance status $=0$ in LI-1 compared with VALOR (20 vs $42 \%$ ), and LI-1 allowed four patients with WHO performance status $\geq 3$ (none in VALOR). The median age of the patients in LI-1 was higher than those in VALOR (75 years for all four cohorts in LI-1 vs 63 and 64 years for the two cohorts in VALOR), and LI-1 included patients ranging in ages from 60 years and up, whereas VALOR included younger patients ranging from 18 years and up. Both used cytarabine; however, dosing regimens were different, with LI-1 using low-dose sc. cytarabine $20 \mathrm{mg}$ twice daily for 10 days and VALOR administering an intermediate-dose cytarabine of $1 \mathrm{~g} / \mathrm{m}^{2}$ iv. for 5 days. Dosing of vosaroxin was lower in LI-1 for the first two cycles (72 vs $90 \mathrm{mg} / \mathrm{m}^{2}$ in VALOR) and nearly equivalent in subsequent cycles ( 72 vs $70 \mathrm{mg} / \mathrm{m}^{2}$ in VALOR). The differences in patient populations, numbers of patients, ages and level of fitness, as well as differences in therapy dosing, necessitate that the two studies' results be assessed independently. In summary, LI-1 tested a lower number of lessfit patients up front with lower doses of vosaroxin with or without cytarabine, and VALOR tested a larger number of fit relapsed/refractory patients with a vosaroxin regimen containing significantly higher doses of therapy. In the latter setting, some older patients and those in early relapse or with refractory disease-derived survival benefit with the addition of vosaroxin.

\section{Patients benefiting from vosaroxin: considerations moving forward}

The cache of new therapies being tested in AML is driven by the persisting need for more effective 
Table 2. Comparison of randomized trials: LI-1 (vosaroxin) and VALOR.

\begin{tabular}{|c|c|c|}
\hline Parameter & LI-1 (vosaroxin) & VALOR \\
\hline Location of trial & UK & International \\
\hline Total number of patients & 208 & 711 \\
\hline Patients receiving vosaroxin, $\mathrm{n}(\%)$ & $106(51)$ & $356(50)$ \\
\hline \multicolumn{3}{|l|}{ Patient characteristics: } \\
\hline - Median age (years), all arms (range) & 75 years $(60-91)$ & $63-64$ years $(18-82)$ \\
\hline - Suitable for intensive therapy allowed? & No & Yes \\
\hline \multicolumn{3}{|l|}{ PS: } \\
\hline - Measure used & WHO & ECOG \\
\hline - PS range & $0-4$ & $0-2$ \\
\hline \multicolumn{3}{|l|}{ - Patients enrolled with PS, n (\%): } \\
\hline$\cdot 0$ & $41(20)$ & $299(42)$ \\
\hline \multicolumn{3}{|l|}{ Cytogenetics: } \\
\hline - Metric used & MRC & NCCN \\
\hline \multicolumn{3}{|l|}{ - Patients in risk stratification, $\mathrm{n}(\%)$ : } \\
\hline - Favorable & $4(2)$ & $16(2)$ \\
\hline - Intermediate & $109(52)$ & $330(46)$ \\
\hline - Adverse/unfavorable & $32(15)$ & $133(9)$ \\
\hline • Unknown & $63(30)$ & $232(33)$ \\
\hline \multicolumn{3}{|l|}{ Therapy: } \\
\hline - Cytarabine dose level & Low & Intermediate \\
\hline - Cytarabine dose, specific & 20 mg sc. b.i.d. days $1-10$ & $1 \mathrm{~g} / \mathrm{m}^{2}$ iv. days $1-5$ \\
\hline
\end{tabular}

treatments. A high proportion of relapse occurs with the current standard of care. Few chemotherapeutics have been developed over the past decades for AML. The success of such therapies depends on two factors: whether it provides improvement over current outcomes, and whether its toxicity profile is superior to current therapies while maintaining effective AML-cell kill. Because of multiple apparent genetic routes in the development of AML [20], personalized therapy designed for baseline disease characteristics is a worthwhile approach. Although there is a trend away from the use of blanket cytotoxic antineoplastic drugs in favor of targeted and immune-based therapies with greater specificity for cancer cells, chemotherapy still remains a mainstay of treatment for AML. Cures to date have been primarily the result of myeloablative therapy. Moreover, there endures a strong need for cytoreductive therapies that are useful in AML patients or AML subsets in combination with newer approaches. Chemotherapy will continue to serve a critical role as debulking agents for the foreseeable future.

AML patients $\geq 60$ years have a particularly poor prognosis, which is undoubtedly a function of both the disease biology in this age group, in addition to the poor tolerability of intense cytotoxic therapies. The additive improvement in survival seen for some patients receiving vosaroxin should not be underestimated. Vosaroxin is associated with fewer potential side effects when compared with anthracyclines, especially with respect to cardiac toxicity. It may be advantageous over idarubicin or daunorubicin because of better tolerance among older AML patients. Vosaroxin plus cytarabine combinations may be an option in patients who cannot tolerate more intense regimens and combinations, or who have progressed after receiving anthracyclines. Further, vosaroxin possess two properties not present in other topoisomerase II inhibitors. 
First, it induces apoptosis in a TP53-independent manner, highlighting its potential usefulness in TP53-mutated AML or AML with chromosome $17 \mathrm{p}$ loss, both of which are thought to be poorly responsive to current chemotherapy [21]. Therapyrelated AML cases, which are also difficult to treat, are enriched for TP53 mutations [22,23]. Second, vosaroxin is not a substrate for the multidrug efflux P-glycoprotein pathway, and so it bypasses this AML resistance mechanism unlike the anthracycline antibiotics $[9,10]$.

Vosaroxin is a reasonable consideration for relapsed/refractory AML patients $\geq 60$ years based on VALOR data. Any relapsed/refractory patient who has already received or may not be fit for anthracycline-based regimens (e.g., due to cardiac risk) or high-dose cytarabine may derive benefit from the addition of vosaroxin to intermediate-dose cytarabine. Vosaroxin is currently under consideration for approval in Europe and is undergoing continued evaluation prior to application for approval in the USA. Vosaroxin may eventually provide an option when considering further lines of chemotherapy, given it is an effective cytotoxic agent with limited toxicity.

It is possible that certain biological subsets of AML may respond better to vosaroxin, and these subsets may be identifiable. It is conceivable that elderly relapsed/refractory AML patients may be enriched for a type of AML with this distinct biology [24]. In VALOR, CR + CRi + CRp rates for patients receiving vosaroxin were comparable between patients younger than 60 years and those of 60 years and older (35 vs $38 \%$, respectively). For both groups, this response rate was significantly higher than equivalently aged patients receiving cytarabine alone $(\mathrm{p}=0.04$ for those $<60$ years and $p<0.0001$ for those $\geq 60$ years). However, a significant overall survival benefit was noted only for patients of 60 years and older who received vosaroxin. Although no survival benefit was detected in younger patients who received vosaroxin, the increased $\mathrm{CR}+\mathrm{CRi}+\mathrm{CRp}$ rate with the addition of vosaroxin denotes a potential therapeutic opportunity and/or benefit in an unidentified subfraction of AML. Future research will focus on better identifying best responders to vosaroxin combinations.

\section{Future combinatorial strategies}

An incremental elevation of the horizontal tail of a Kaplan-Meier survival curve indicates piecemeal progress in the treatment of cancers. The accretion of a number of modest elevations is one path to breakthrough status in AML. Vosaroxin produces an improvement over the therapeutic approach of using cytarabine alone in some subsets of patients. Although it may be incorporated into treatment practice for patients older than 60 years with relapsed/refractory disease, research has yet to determine whether it can provide additional benefit in combination with other experimental agents. Rational combinations and sequential applications of concomitant therapies will be investigated next. Current investigations include combining vosaroxin with hypomethylating agents for myelodysplastic syndrome and AML, and with cytarabine as front-line therapy in AML patients 60 years and older (Clinicaltrials.gov identifiers NCT01913951, NCT01980056, NCT01893320 and NCT02485353).

Although the mechanism by which vosaroxin intercalates DNA and poisons topoisomerase II is specific and elegant, vosaroxin may still be considered as a cytotoxic chemotherapeutic. Such agents continue to serve a central role in the therapy of AML as the cornerstone of cytoreduction. For these agents, maximizing leukemia cell killing within nontoxic dose limitations is paramount. Theoretically, combinatorial approaches may target cells in various stages of the cell cycle, reduce resistance, provide alternate methods of cell killing for different AML subpopulations and/or target cells in different microenvironment niches. Vosaroxin is a unique quinolone topoisomerase II inhibitor and exhibits distinct apoptotic action by challenging AML cells differently than other therapeutics. As new therapies are added to the armamentarium to treat AML, targeting leukemic cells with mechanistically diverse modalities is advantageous. Therapeutic approaches that alter the methylation status, target stem-cell subpopulations or train an immune response against leukemic cells will all likely be combined with cytoreductive agents. Vosaroxin adds a novel component with a favorable safety profile to the field of cytotoxic players.

\section{Conclusion}

Although significant strides are made in the treatment of AML, it is of great importance that vosaroxin was tested rigorously in a multinational collaboration large enough to perform prespecified subset analyses. This approach identified superior outcomes with the addition of vosaroxin in certain patients with AML. With an established unique mode of action, Vosaroxin 
will likely be considered in novel combinations of active agents against AML. It is notable for its activity in older patients with relapsed/refractory AML, who may be difficult to treat due to resistant disease and decreased tolerance for more intensive therapies. Future trials will continue to define its role as part of the increasingly multimodal strategy against AML.

\section{Disclosure}

In addition to the peer-reviewed process, with the authors consent, the manufacturer of the product discussed in this article was given the opportunity to review the manuscript for factual accuracy. Changes were made by the authors at their discretion and based on scientific or editorial merit only. The authors maintained full control over the manuscript, including content, wording and conclusions.

Financial \& competing interests disclosure

$F$ Ravandi has received research funding and Honoraria from Sunesis and has been the member of advisory boards with Sunesis. The authors have no other relevant affiliations or financial involvement with any organization or entity with a financial interest in or financial conflict with the subject matter or materials discussed in the manuscript apart from those disclosed.

No writing assistance was utilized in the production of this manuscript.

\section{EXECUTIVE SUMMARY}

\section{Pharmacology of vosaroxin}

- Primary mechanism of action is intercalation into DNA during G2/M in GC-rich areas and inhibition of topoisomerase II to induce TP53-independent apoptosis.

- In pharmacokinetics studies, older patients metabolized vosaroxin similarly to younger patients, and early clinical data showed a favorable safety profile.

\section{Clinical studies of vosaroxin plus cytarabine}

- Phase I and II single-arm studies of vosaroxin and vosaroxin plus cytarabine demonstrated activity and tolerability in acute myeloid leukemia patients.

- Recent Phase III VALOR trial demonstrated that adding vosaroxin to cytarabine led to superior overall survival in relapsed/refractory patients $\geq 60$ years and early relapsed/refractory patients.

\section{Combinations with other agents}

- With activity in acute myeloid leukemia, favorable toxicity profile, tolerability among older patients and incremental survival benefit in subsets, vosaroxin remains an attractive choice for rationally designed combinatorial strategies.

\section{References}

Papers of special note have been highlighted as:

- of interest; $\bullet \bullet$ of considerable interest

1 Sissi C, Palumbo M. The quinolone family: from antibacterial to anticancer agents. Curr. Med. Chem. Anticancer Agents 3(6), 439-450 (2003).

2 Hawtin RE, Stockett DE, Byl JAW et al. Voreloxin is an anticancer quinolone derivative that intercalates DNA and poisons topoisomerase II. PLoS ONE 5(4), e10186 (2010).

- Laboratory studies deciphering fundamental mechanisms of vosaroxin's action.

3 Walsby EJ, Coles SJ, Knapper S, Burnett AK. The topoisomerase II inhibitor voreloxin causes cell cycle arrest and apoptosis in myeloid leukemia cells and acts in synergy with cytarabine. Haematologica 96(3), 393-399 (2011).
- Laboratory studies detailing vosaroxin's action and demonstrating synergism with cytarabine against leukemia cells.

4 Siegel RL, Miller KD, Jemal A. Cancer statistics, 2016. CA Cancer J. Clin. 66(1), 7-30 (2016).

5 Estey E. Acute myeloid leukemia: 2016 update on risk-stratification and management. Am. J. Hematol. 91(8), 824-846 (2016).

6 Ravandi F, Ritchie EK, Sayar H et al. Vosaroxin plus cytarabine versus placebo plus cytarabine in patients with first relapsed or refractory acute myeloid leukaemia (VALOR): a randomised, controlled, double-blind, multinational, Phase 3 study. Lancet Oncol. 16(9), 1025-1036 (2015).

-• Describes the largest Phase III study evaluating vosaroxin plus cytarabine in acute myeloid leukemia (AML).
7 Richter SN, Giaretta G, Comuzzi V et al. Hot-spot consensus of fluoroquinolonemediated DNA cleavage by Gram-negative and Gram-positive type II DNA topoisomerases. Nucleic Acids Res. 35(18), 6075-6085 (2007)

8 Hawtin RE, Stockett DE, Wong OK, Lundin C, Helleday T, Fox JA. Homologous recombination repair is essential for repair of vosaroxin-induced DNA double-strand breaks. Oncotarget 1(7), 606-619 (2010).

- Laboratory studies investigating an aspect of vosaroxin's action on DNA.

9 Shaffer BC, Gillet JP, Patel C, Baer MR, Bates SE, Gottesman MM. Drug resistance: still a daunting challenge to the successful treatment of AML. Drug Resist. Updates 15(1-2), 62-69 (2012).

10 Freeman C, Keane N, Swords R, Giles F. Vosaroxin: a new valuable tool with the 
potential to replace anthracyclines in the treatment of AML? Expert Opin.

Pharmacother. 14(10), 1417-1427 (2013).

11 Evanchik MJ, Allen D, Yoburn JC, Silverman JA, Hoch U. Metabolism of (+)-1,4-dihydro7-(trans-3-methoxy-4-methylamino-1pyrrolidinyl)-4-oxo-1-(2-thiaz olyl)-1,8naphthyridine-3-carboxylic acid (voreloxin; formerly SNS-595), a novel replicationdependent DNA-damaging agent. Drug Metab. Dispos. 37(3), 594-601 (2009).

12 Lancet JE, Ravandi F, Ricklis RM et al. A Phase Ib study of vosaroxin, an anticancer quinolone derivative, in patients with relapsed or refractory acute leukemia. Leukemia 25(12), 1808-1814 (2011).

13 Advani RH, Hurwitz HI, Gordon MS et al. Voreloxin, a first-in-class anticancer quinolone derivative, in relapsed/refractory solid tumors: a report on two dosing schedules. Clin. Cancer Res. 16(7), 2167-2175 (2010).

14 Krug LM, Crawford J, Ettinger DS et al. Phase II multicenter trial of voreloxin as second-line therapy in chemotherapy-sensitive or refractory small-cell lung cancer. J. Thorac. Oncol. 6(2), 384-386 (2011).

15 Abbas JA, Stuart RK. Vosaroxin: a novel antineoplastic quinolone. Expert Opin. Investig. Drugs 21(8), 1223-1233 (2012).

16 Stuart RK, Cripe LD, Maris MB et al. REVEAL-1, a Phase 2 dose regimen optimization study of vosaroxin in older poor-risk patients with previously untreated acute myeloid leukaemia. Br. J. Haematol. 168(6), 796-805 (2015).

17 Kantarjian H, O’Brien S, Cortes J et al. Results of intensive chemotherapy in 998 patients age 65 years or older with acute myeloid leukemia or high-risk myelodysplastic syndrome: predictive prognostic models for outcome. Cancer 106(5), 1090-1098 (2006).

18 Lancet JE, Roboz GJ, Cripe LD et al. A Phase $1 \mathrm{~b} / 2$ study of vosaroxin in combination with cytarabine in patients with relapsed or refractory acute myeloid leukemia. Haematologica 100 (2), 231-237 (2015).

19 Dennis M, Russell N, Hills RK et al. Vosaroxin and vosaroxin plus low-dose Ara-C (LDAC) vs low-dose Ara-C alone in older patients with acute myeloid leukemia. Blood 125(19), 2923-2932 (2015).

-. Describes one of two Phase III studies to date of vosaroxin with and without cytarabine in AML.

20 Cancer Genome Atlas Research Network. Genomic and epigenomic landscapes of adult de novo acute myeloid leukemia. N. Engl. J. Med. 368(22), 2059-2074 (2013).

21 Kadia TM, Jain P, Ravandi F et al. TP53 mutations in newly diagnosed acute myeloid leukemia: clinicomolecular characteristics, response to therapy, and outcomes.
Cancer doi:10.1002/cncr.30203 (2016) (Epub ahead of print).

-• Largest series to investigate TP53-mutated AML.

22 Kayser S, Dohner K, Krauter J et al. The impact of therapy-related acute myeloid leukemia (AML) on outcome in 2853 adult patients with newly diagnosed AML. Blood 117(7), 2137-2145 (2011).

23 Wong TN, Ramsingh G, Young AL et al. Role of TP53 mutations in the origin and evolution of therapy-related acute myeloid leukaemia. Nature 518(7540), 552-555 (2015).

24 Pollyea DA, Kohrt HE, Medeiros BC. Acute myeloid leukaemia in the elderly: a review. Br. J. Haematol. 152(5), 524-542 (2011).

25 Montalban-Bravo G, Garcia-Manero G. Novel drugs for older patients with acute myeloid leukemia. Leukemia 29(4), 760-769 (2015).

26 Scatena CD, Kumer JL, Arbitrario JP et al. Voreloxin, a first-in-class anticancer quinolone derivative, acts synergistically with cytarabine in vitro and induces bone marrow aplasia in vivo. Cancer Chemother. Pharmacol. 66(5), 881-888 (2010).

27 Appelbaum FR, Gundacker H, Head DR et al. Age and acute myeloid leukemia. Blood 107(9), 3481-3485 (2006). 